\title{
Learning Diagnostic Imaging: 100 Essential Cases
}

R. Ribes, A. Luna, and P.R. Ros, eds.

Berlin, Germany: Springer-Verlag, 2008, 254 pages, $\$ 59.95$

The purpose of this book is to provide a quick introduction to diagnostic imaging for medical students and radiology residents, as well as technicians, physician assistants, and nurses associated with diagnostic imaging. Because the book is divided into subspecialties, it may also serve as a refresher for specialized radiologists who are encountering unexpected findings.

Each of the 10 sections of the book addresses a diagnostic imaging subspecialty. The 10 subspecialties are breast imaging; cardiovascular imaging; thoracic radiology; the gastrointestinal system and disorders of the liver, pancreas, spleen, and biliary system; genitourinary, gynecologic, and obstetric imaging; musculoskeletal imaging; neuroimaging and head and neck imaging; nuclear medicine; pediatric radiology; and ultrasound imaging. After an introduction on the subspecialty, 10 case studies within that subspecialty are presented in a consistent format: case history, pathophysiology, and imaging findings. Given the stated purpose of the book of reaching an audience of medical students and beginning diagnostic imaging residents, the best cases to present in each section are debatable. As a composite, the cases chosen provide a quick and comprehensive overview of radiology. The case selection could present situations that are more commonly encountered in daily practice, such as pneumonia, atelectasis, effusions, ectopic pregnancy, child abuse, peptic ulcer disease, ileus, bowel obstruction, and goiter. Most of the images presented with the cases are of good quality.

One of the best features in each section is the listing of further reading. Appropriate books, Web links, and articles that the reader can pursue for further information are listed. The editors are also commended for the up-to-date, userfriendly reference lists in each section.

The audience can be expected to have limited knowledge of the normal anatomic diagnostic imaging appearance of the selected body parts and to lack familiarity with the terminology of each subspecialty, such as echogenicity, MRI intensity and pulse sequences, and radiopharmaceuticals. It will be difficult for the novice to identify abnormal findings without knowledge of what can be considered normal. Though legends are given for each case, most images are not annotated as to the abnormal findings-a drawback and source of difficulty for the novice. The section on thoracic radiology is in bullet format, which some readers, especially radiology residents, may prefer.

This book serves as a teaching file for beginners in diagnostic imaging but is limited in its comprehensiveness and has built-in difficulties for readers.

\section{Aurelio Matamoros, Jr. M.D. Anderson Cancer Center Houston Texas}

